\title{
ARTICLES
}

\section{Chemical genetics of Plasmodium falciparum}

\author{
W. Armand Guiguemde ${ }^{1}$, Anang A. Shelat ${ }^{1}$, David Bouck ${ }^{1}$, Sandra Duffy ${ }^{2}$, Gregory J. Crowther ${ }^{3}$, Paul H. Davis ${ }^{4}$, \\ David C. Smithson ${ }^{1}$, Michele Connelly ${ }^{1}$, Julie Clark ${ }^{1}$, Fangyi Zhu ${ }^{1}$, María B. Jiménez-Díaz ${ }^{5}$, María S. Martinez ${ }^{5}$, \\ Emily B. Wilson ${ }^{6}$, Abhai K. Tripathi ${ }^{7}$, Jiri Gut ${ }^{8}$, Elizabeth R. Sharlow ${ }^{9}$, Ian Bathurst ${ }^{10}$, Farah El Mazouni ${ }^{11}$, \\ Joseph W. Fowble ${ }^{12}$, Isaac Forquer ${ }^{13}$, Paula L. McGinley ${ }^{14}$, Steve Castro ${ }^{14}$, Iñigo Angulo-Barturen ${ }^{5}$, Santiago Ferrer ${ }^{5}$, \\ Philip J. Rosenthal ${ }^{8}$, Joseph L. DeRisi ${ }^{6}$, David J. Sullivan Jr ${ }^{7}$, John S. Lazo ${ }^{9}$, David S. Roos ${ }^{4}$, Michael K. Riscoe ${ }^{13}$, \\ Margaret A. Phillips ${ }^{11}$, Pradipsinh K. Rathod ${ }^{12}$, Wesley C. Van Voorhis ${ }^{3}$, Vicky M. Avery ${ }^{2}$ \& R. Kiplin Guy ${ }^{1}$
}

\begin{abstract}
Malaria caused by Plasmodium falciparum is a disease that is responsible for 880,000 deaths per year worldwide. Vaccine development has proved difficult and resistance has emerged for most antimalarial drugs. To discover new antimalarial chemotypes, we have used a phenotypic forward chemical genetic approach to assay 309,474 chemicals. Here we disclose structures and biological activity of the entire library-many of which showed potent in vitro activity against drug-resistant $P$. falciparum strains-and detailed profiling of 172 representative candidates. A reverse chemical genetic study identified 19 new inhibitors of 4 validated drug targets and 15 novel binders among 61 malarial proteins. Phylochemogenetic profiling in several organisms revealed similarities between Toxoplasma gondii and mammalian cell lines and dissimilarities between $P$. falciparum and related protozoans. One exemplar compound displayed efficacy in a murine model. Our findings provide the scientific community with new starting points for malaria drug discovery.
\end{abstract}

The widespread resistance of $P$. falciparum to many antimalarial drugs, the dependence of all new drug combinations on artemisinins (for which resistance may have emerged $)^{1,2}$, and new efforts to eradicate malaria all drive the need to develop new, effective and affordable antimalarial drugs ${ }^{3}$. Although our understanding of the parasite's biology has increased with the sequencing of the Plasmodium genome ${ }^{4}$ and the development of new technologies to study resistance acquisition $^{5-7}$, few new drug targets or classes of drugs have been clinically validated ${ }^{8}$. The lack of publicly accessible antimalarial chemotypes with differing modes of action has significantly hindered efforts to discover and develop new drugs ${ }^{9}$. To address this urgent need, we have developed a forward chemical genetic approach to identify novel antimalarials (Supplementary Fig. 1).

\section{The forward chemical genetic screen}

A library containing 309,474 unique compounds, designed at the scaffold level to provide diverse, comprehensive coverage of bioactive space $^{10,11}$, was screened against Plasmodium falciparum strain 3D7 at a fixed concentration of $7 \mu \mathrm{M}$ (Supplementary Information) ${ }^{12}$. Fidelity of the assay was examined by receiver operator characteristic (ROC) analysis and other metrics (Supplementary Figs 2 and 3), demonstrating good discriminatory power (area under the curve $\sim 0.85$ ) and indicating that a cutoff of $\geq 80 \%$ activity would retain most of the true positives. The strength of the assay was further determined by testing a set of bioactive compounds including known antimalarials, all of which were re-identified (Supplementary Table 3), demonstrating that the method was very likely to identify any molecule acting by a known mechanism. The primary screen gave approximately 1,300 hits with activity $>80 \%$. These compounds were serially diluted and tested against both the chloroquine-sensitive 3D7 strain and the chloroquine-resistant K1 strain, giving 1,134 validated hits that had saturated dose-response curves. Chemical structure analysis of validated hits by topology mapping and clustering ${ }^{10}$ revealed a wide distribution of chemotypes in the active chemical space, with several displaying promising structure-activity relationships (Fig. 1). Although all known antimalarial scaffolds (aminoquinolines, quinolones, bis-amidines) present in the screening collection were identified, providing positive controls for the screen, most of the chemotypes identified were new. A total of 561 of the validated hits had halfmaximum effective concentration $\left(\mathrm{EC}_{50}\right)$ values $\leq 2 \mu \mathrm{M}$ against either $3 \mathrm{D} 7$ or $\mathrm{K} 1$ and a therapeutic window $\geq 10$-fold against two mammalian cell lines (HepG2 and BJ). From this set, 228 structurally distinct, pure compounds were re-purchased in powder form for further studies. Antimalarial potencies of $\sim 75 \%$ of these compounds (172) were reconfirmed to within tenfold (Bland-Altman analysis, Supplementary Fig. 4) by three laboratories using distinct methods providing the crossvalidated hit set used for all subsequent experiments.

\section{Combination with antimalarial drugs}

Owing to rapid resistance acquisition, the World Health Organization (WHO) recommends combination therapy ${ }^{13}$. The agonistic and antagonistic synergies of the cross-validated set were therefore quantified by measuring $\mathrm{EC}_{50}$ shifts in the presence of a fixed fraction of potency $\left(\mathrm{EC}_{10}\right)$ concentration of chloroquine, mefloquine, artemisinin and atovaquone. Most cross-validated compounds were additive in effect or had minor synergies with existing drugs. Two classes demonstrated strong synergies ( $\mathrm{EC}_{50}$ values reduced by $\geq 10$-fold): the diaminonaphthoquinones with artemisinin, and the dihydropyridines 


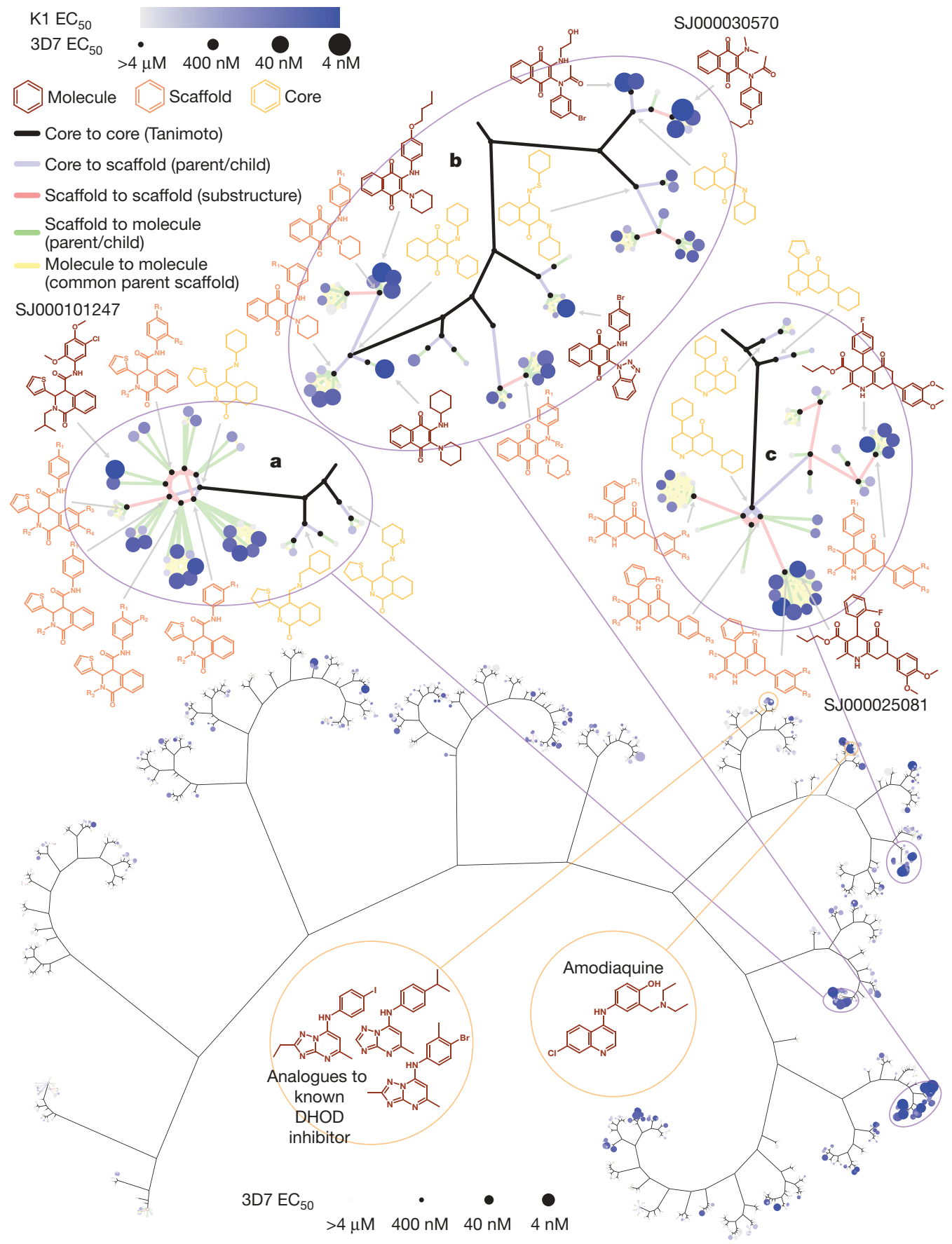

Figure 1 | Chemical structure network graph and antimalarial potencies of the 1,300 primary screen hits. Topologically similar molecules cluster together in the branches of the network. To construct the graph, molecules were first abstracted to scaffolds and then further to cores using the Murcko algorithm ${ }^{10}$. Each of these structural entities is represented as a node, and nodes are connected via edges according to topological relationships with closeness being defined using the Tanimoto coefficient. Molecular nodes are coded to reflect potency against $P$. falciparum strains K1 (low, white; high, blue) and 3D7 (low, small; high, large). The highly branched structure of the

with mefloquine (Fig. 2). One diaminonaphthoquinone and a cycloguanil analogue displayed antagonism with chloroquine and mefloquine, respectively.

\section{Reverse chemical genetics}

The advantages of phenotypic screens for the identification of novel chemotypes are that no a priori assumptions are made concerning drug targets and that active compounds inherently have cellular bioavailability. Because insight into the mechanism of action is helpful for full network graph (bottom half of the figure) indicates that the 1,300 compounds are organized into clusters of clusters: cores are well sampled by multiple scaffolds, and the cores themselves are grouped into families of related chemotypes. Previously reported antimalarial compounds are highlighted in the lower centre. The top half of the figure provides greater detail on three potent chemotypes with well-developed structure activity relationships: $\mathbf{a}$, tetrahydroisoquinoline; $\mathbf{b}$, diaminonaphthoquinone; c, dihydropyridine. Data are in Supplementary Information.

drug development, we also investigated the interaction of the crossvalidated set with 66 potential targets using enzyme inhibition assays and thermal melt shift assays (to detect binding).

Three high-priority, well characterized biological targets were evaluated in activity assays (Fig. 3, left): P. falciparum dihydroorotate dehydrogenase (PfDHOD), haemozoin formation and P. falciparum falcipain-2 (PfFP-2). PfDHOD catalyses the oxidation of dihydroorotate to orotate in de novo pyrimidine biosynthesis, which is essential for parasite viability ${ }^{14,15}$. Three compounds inhibited this enzyme: two 

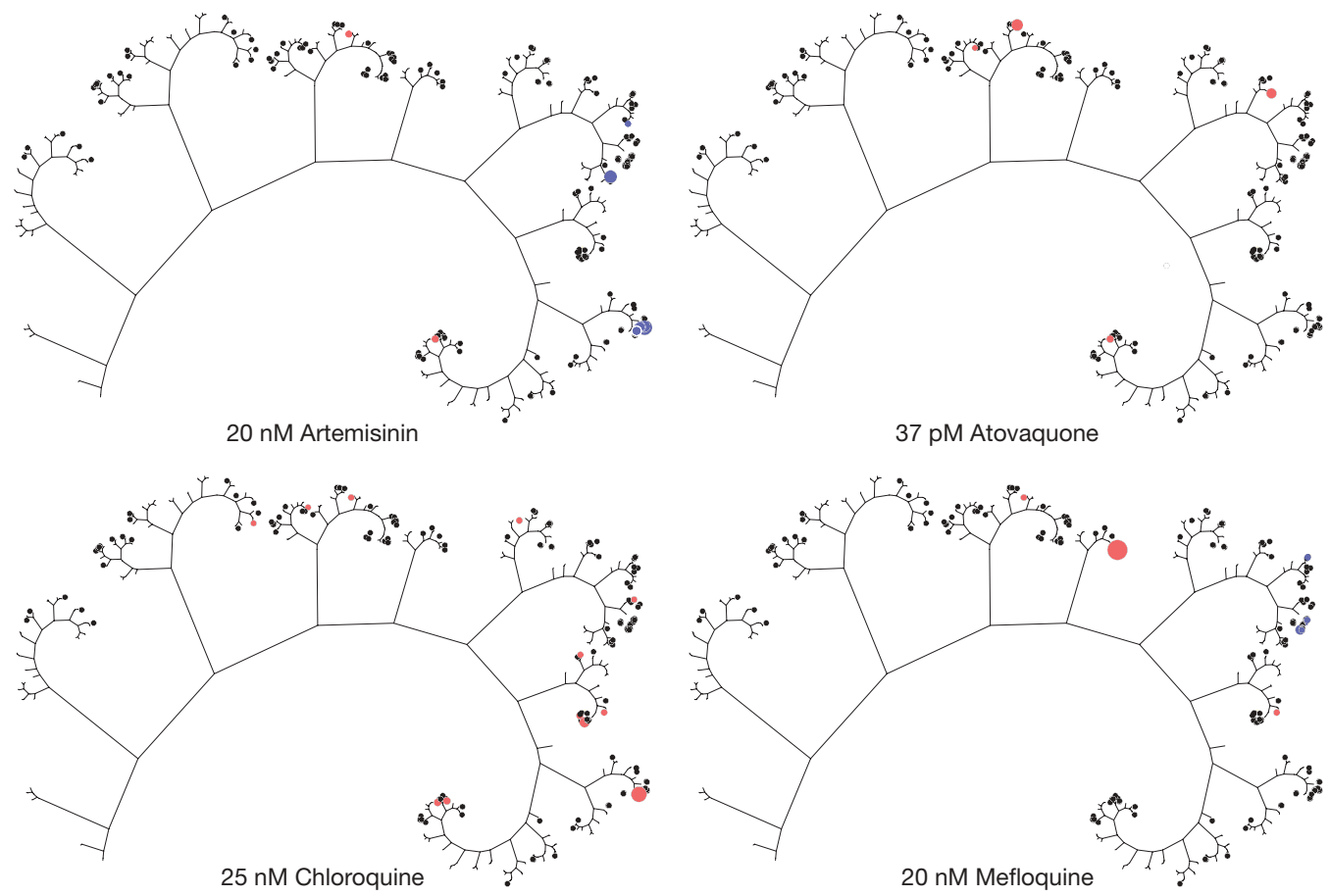

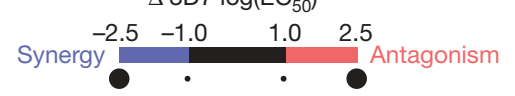

Figure 2 | Reduced representation of the network map showing synergistic activities with clinically relevant antimalarials. The size of the nodes reflects the magnitude of the logarithmic difference between $\mathrm{EC}_{50}$ in the presence and absence of $\mathrm{EC}_{10}$ of exemplar antimalarial drugs. Absolute

triazolopyrimidines, structurally related to known PfDHOD inhibitors with comparable potencies ${ }^{14}$, and a dihydropyridine, structurally related to the calcium blocker felodipine. The potency of these compounds against PfDHOD strongly correlated with their antimalarial activities (Supplementary Table 5). Furthermore, these compounds were inactive against transgenic parasites expressing Saccharomyces cerevisiae dihydroorotate dehydrogenase (Supplementary Table 6). Next, haemozoin formation inhibition was investigated. The parasite digests host haemoglobin to provide amino acids, detoxifying the resulting haem molecules by conversion to a crystallized form known as haemozoin. Haem detoxification is believed to be the target of many antimalarial drugs ${ }^{16}$. Twelve compounds showed appreciable efficacy in an in vitro haemozoin formation assay ${ }^{17}$, including analogues of quinazoline, benzofuran, benzimidazole and carbazole as well as amodiaquine, a known haemozoin formation inhibitor present in our library. The correlation between enzyme inhibitory potency and

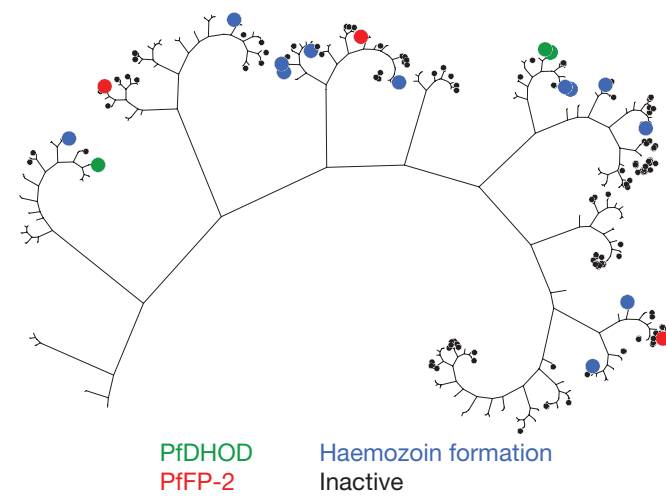

Figure 3 | Reduced representation of the network map showing the interaction of the cross-validated hits with potential biological targets. The network map on the left displays compounds targeting well-validated protein targets as measured in inhibition assays $\left(\mathrm{EC}_{50} \leq 15 \mu \mathrm{M}\right)$. The map differences less than one log unit were not considered significant. Synergistic and antagonistic compounds are uniformly colour-coded blue and red, respectively. Highly synergistic compounds can be seen with artemisinin and mefloquine. Data are in Supplementary Information.

antimalarial potency was similar to that displayed by the positive controls quinine and amodiaquine (Supplementary Table 5). The third enzyme assayed was PfFP-2, which has a critical role in haemoglobin degradation ${ }^{18}$. Falcipains are redundant in P. falciparum, with four known homologues including two (falcipain-2 and falcipain-3) that seem to have key roles in erythrocytic stage parasites ${ }^{19}$. Three weakly active PfFP-2 inhibitors were identified. Thus, 19 compounds (11\%) were inhibitors of validated antimalarial targets.

To expand the pool of potential targets, the compounds were tested for binding to 61 recombinant malarial proteins (95\% purity or better after affinity and size exclusion chromatography; Supplementary Table 1) in a thermal melt shift assay ${ }^{20}$. Fifteen compounds displayed reproducible thermal shifts with seven malarial proteins (Fig. 3, right; dissociation constant $\left(K_{\mathrm{d}}\right)$ values in Supplementary Table 2): 6-phosphogluconolactonase, 6-pyruvoyltetrahydropterin synthase, choline kinase, D-ribulose-5-phosphate 3-epimerase, dUTPase, glycogen

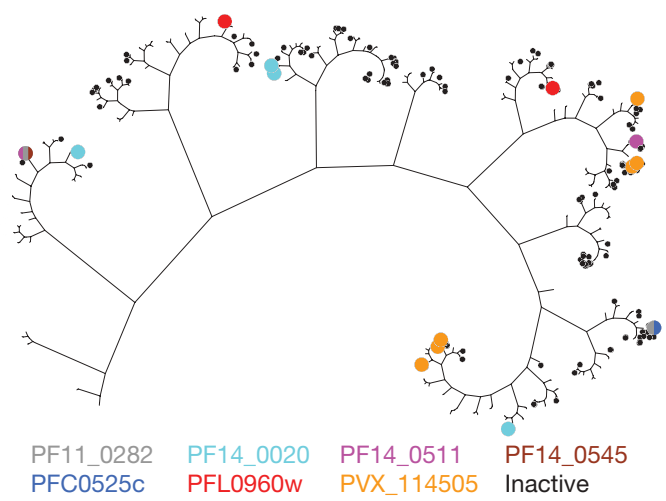

on the right shows compounds that bind to purified malarial proteins according to thermal melt shift experiments. The size of nodes representing active or binding compounds is increased for clarity. Data are in Supplementary Information. 
synthase kinase 3 and thioredoxin. Two compounds bound multiple proteins. Two out of the seven proteins are in essential malarial pathways: phosphatidylcholine synthesis ${ }^{21}$ (choline kinase) and redox metabolism $^{22}$ (thioredoxin). The remaining five protein targets potentially represent novel antimalarial drug targets.

\section{The potential for cross-resistance}

To evaluate the potential for cross-resistance with existing drugs, the cross-validated compounds were tested against a panel of $P$. falciparum strains with different chemosensitivities to known antimalarials, including strains 3D7 (chloroquine sensitive), K1, W2, V1/S and Dd2 (all resistant to both chloroquine and to antifolates), and SB-A6 and D10_yDHOD (both chloroquine sensitive and atovaquone resistant). All strains were profiled for sensitivity to a set of antimalarial drugs to normalize activity (Supplementary Table 3). A total of 58 cross-validated compounds displayed similar potencies $\left(\mathrm{EC}_{50}\right.$ shift $\leq 3$-fold) against 3D7, K1, V1/S and SB-A6, indicating that these compounds do not share mechanisms of resistance with chloroquine, atovaquone or sulphadoxine/pyrimethamine. A subset of the $172 \mathrm{com}$ pounds that were inactive against drug-resistant $P$. falciparum strains with known mutations in target proteins were tested against 3D7 dihydrofolate reductase and Plasmodium yoelii cytochrome $b c_{1}$ complex in biochemical assays. Two inhibitors were identified for each protein (Supplementary Tables 5 and 6).

\section{Phylochemogenetic profiling}

To understand relationships between chemical sensitivity of Plasmodium and related parasites, the cross-validated set was tested against three additional protozoan parasite species-Toxoplasma gondii, which belongs to the same phylum as Plasmodium (Apicomplexa), and Leishmania major and Trypanosoma brucei, which are both Kinetoplastida, unrelated to the Apicomplexa-and an expanded panel of human cell lines including a Burkitt's lymphoma line (Raji) and embryonic kidney fibroblast cells (HEK293). Phylogenetic criteria predict that chemical sensitivity should correlate with evolutionary history, due to homology between key protein targets, as is known to be the case for many antiparasitic drugs ${ }^{23,24}$. Although a few compounds showed activity in other parasites, most were highly selective for Plasmodium (Fig. 4), whereas Toxoplasma exhibited a chemosensitivity pattern more similar to human cell lines. Similarly, the highly potent anti-leishmanial benzothiazoles were only weakly active against the related Trypanosoma. These findings indicate that chemical sensitivity of pathogens is regulated by a combination of pathogen genetics, physiology and relationships to host and vector species in vivo.

\section{Early leads for drug development}

To understand the potential for development of the novel chemotypes, the pharmacokinetic properties of the cross-validated set were assessed. The majority are reasonably drug-like, with $78 \%$ of compounds having no violations of the Lipinski rule of five, a well validated predictor of oral bioavailability, and 99\% having one or fewer violations ${ }^{25}$. Within the cross-validated set were embedded three chemical series that had multiple members that together gave structure-activity relationships that spanned 1,000-fold potency differences, had consistent activity in drug-resistant strains, had very good cellular therapeutic windows, and had at least one member with an $\mathrm{EC}_{50}$ more potent than $50 \mathrm{nM}$. An exemplar compound was selected from each series and fully profiled using standard models of in vitro and in vivo adsorption, distribution, metabolism and toxicity (Supplementary Table 7). Each possessed reasonable characteristics for developable hits; indeed, each comes close to passing Medicines for Malaria Venture (MMV) criteria for 'late leads'. The compound from these exemplars with the best pharmacokinetic profile was further evaluated to measure in vivo antimalarial activity and displayed efficacy in a murine malaria model infected with $P$. yoelii. A twice-daily administration of $100 \mathrm{mg} \mathrm{kg}^{-1}$ for 3 days resulted in a $90 \%$ suppression of the parasitaemia (Supplementary Fig. 5). Although it is not suggested that

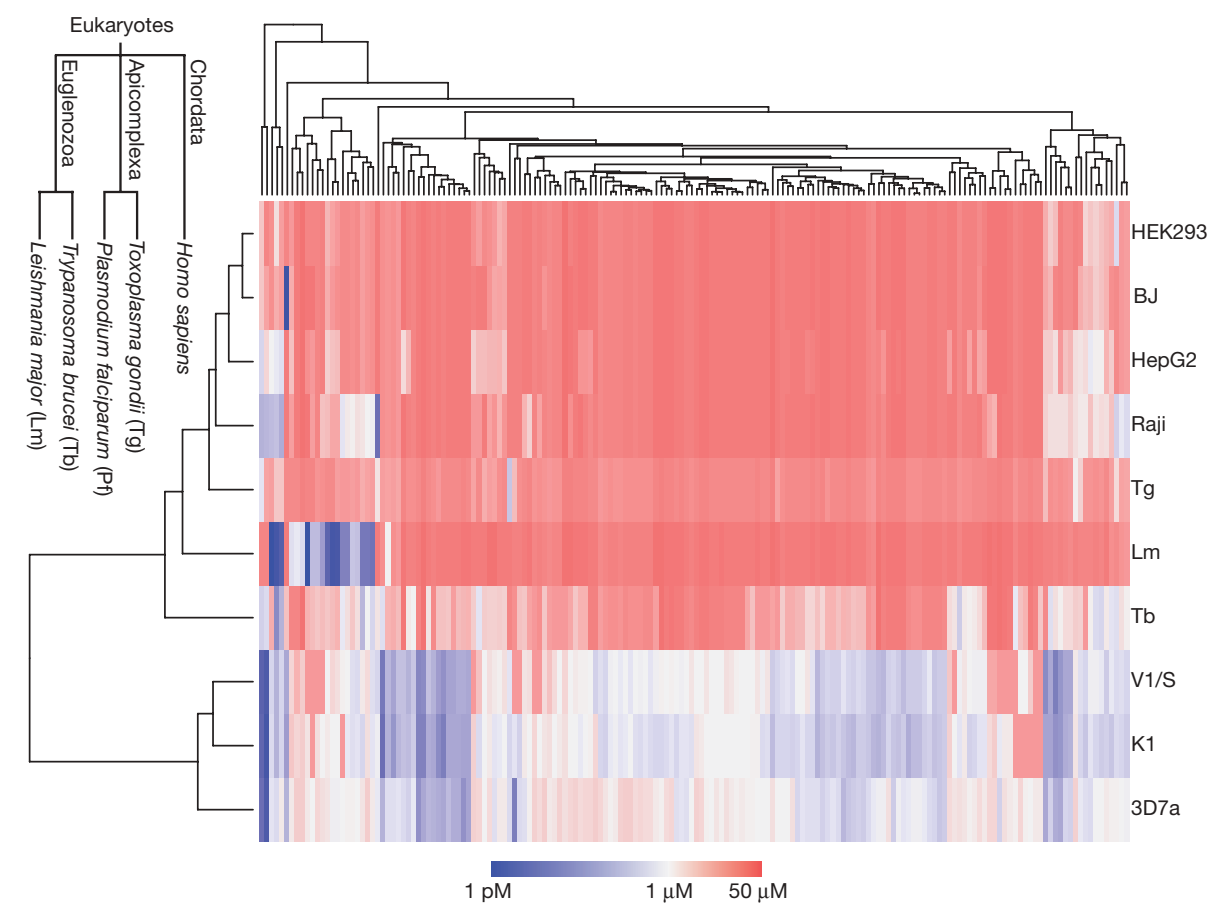

Figure 4 | Phylochemogenetic profiling. Phylochemogenetic analysis of the cross-validated compounds using a two-way hierarchical clustering of growth inhibition against $P$. falciparum strains (3D7, K1, V1/S), other eukaryotic parasites (Toxoplasma gondii $(\mathrm{Tg})$, Trypanosoma brucei $(\mathrm{Tb})$, Leishmania major (Lm)) and human cell lines (HEK293, BJ, Raji and HepG2). Columns represent single compounds and are clustered according to potency against the cell lines and organisms. Neighbouring compounds share a similar potency spectrum. Rows represent a single cell line or organism and are clustered according to their chemosensitivity to the compounds in the study. A phylogenetic tree of the organisms in this study is provided for reference. Note that despite the many known examples of taxonomically conserved pathways, on a global level, phylogeny is a poor predictor of chemical sensitivity profiles: Toxoplasma responses more closely parallel human than their evolutionary siblings, Plasmodium. Data are in Supplementary Information. 
any of the compounds discussed herein are bone fide preclinical candidates, all provide reasonable starting points for drug development.

\section{Conclusions}

Drug therapy remains a key component in controlling malaria. Current challenges of rapid acquisition of resistance, cross-resistance and dependence on a limited number of chemical classes of antimalarials highlight the need to enhance our understanding of the 'chemical space' that can be brought to bear on malaria treatment. Solving this problem requires understanding the relationships between the structures of compounds active against malaria parasites, and their potency, selectivity and targets. We have identified a number of novel compounds and defined these relationships. We expect that these findings will provide novel paths for drug development and hope that making this set of well characterized, non-proprietary lead antimalarials publicly available to the global research community will help to reinvigorate drug discovery for malaria.

\section{METHODS SUMMARY}

The primary screen was carried out by comparing quantities of DNA in treated and control cultures of Plasmodium falciparum in human erythrocytes after $72 \mathrm{~h}$ incubation with a fixed concentration of $7 \mu \mathrm{M}$ of the test compounds. The secondary potency determination was made by using the same assay in a dose-response mode with 10 concentrations varying from $10 \mu \mathrm{M}$ to $5 \mathrm{nM}$. Chemical sensitivities of the human cell lines and T. brucei were determined by measuring their ATP content (Cell Titer Glo, Promega). T. gondii parasites expressing luciferase were cultured and drug sensitivity was determined by luminescence; L. major promastigote drug susceptibility was tested using a metabolic function assay (Alamar Blue, Promega). Chemicals were assayed for haemozoin formation ${ }^{17}$ and PfDHOD ${ }^{15}$ and PfFP- $2^{26}$ inhibitory activities based on previously described methods. Thermal shift assays were done at compound concentrations of $25 \mu \mathrm{M}$ and protein concentrations of $100 \mu \mathrm{g} \mathrm{ml}^{-1}$. All data processing and visualization, and chemical similarity and substructure analysis, was performed using custom programs written in the Pipeline Pilot platform (Accelrys, v.7.0.1) and the R program ${ }^{27}$. A complete description of the methods can be found in Supplementary Information.

The Supplementary Information provides a summary of all relevant data arising from the phenotypic screen and all secondary screens including relevant diagnostics and details about the following: cell-based, enzyme and thermal shift screens, data processing, Bland-Altman analysis, and the algorithm to generate the chemical structure network graph. Chemical structures annotated with assay data and high-resolution PDFs of the figures may be downloaded from http:// www.stjuderesearch.org/guy/data/malaria/.

\section{Received 20 January; accepted 21 April 2010.}

1. Wongsrichanalai, C. \& Meshnick, S. R. Declining artesunate-mefloquine efficacy against falciparum malaria on the Cambodia-Thailand border. Emerg. Infect. Dis. 14, 716-719 (2008).

2. Dondorp, A. M. et al. Artemisinin resistance in Plasmodium falciparum malaria. N. Engl. J. Med. 361, 455-467 (2009)

3. Ridley, R. G. Medical need, scientific opportunity and the drive for antimalarial drugs. Nature 415, 686-693 (2002).

4. Kissinger, J. C. et al. The Plasmodium genome database. Nature 419, 490-492 (2002)

5. Baniecki, M. L., Wirth, D. F. \& Clardy, J. High-throughput Plasmodium falciparum growth assay for malaria drug discovery. Antimicrob. Agents Chemother. 51, 716-723 (2007).

6. Plouffe, D. et al. In silico activity profiling reveals the mechanism of action of antimalarials discovered in a high-throughput screen. Proc. Natl Acad. Sci. USA 105, 9059-9064 (2008).

7. Weisman, J. L. et al. Searching for new antimalarial therapeutics amongst known drugs. Chem. Biol. Drug Des. 67, 409-416 (2006)

8. Wells, T. N., Alonso, P. L. \& Gutteridge, W. E. New medicines to improve control and contribute to the eradication of malaria. Nature Rev. Drug Discov. 8, 879-891 (2009).

9. Munos, B. Can open-source R\&D reinvigorate drug research? Nature Rev. Drug Discov. 5, 723-729 (2006).

10. Shelat, A. A.\& Guy, R. K. Scaffold composition and biological relevance of screening libraries. Nature Chem. Biol. 3, 442-446 (2007).

11. Shelat, A. A. \& Guy, R. K. The interdependence between screening methods and screening libraries. Curr. Opin. Chem. Biol. 11, 244-251 (2007).

12. Smilkstein, M. et al. Simple and inexpensive fluorescence-based technique for high-throughput antimalarial drug screening. Antimicrob. Agents Chemother. 48 , 1803-1806 (2004)
13. Cibulskis, R. E. et al. Estimating trends in the burden of malaria at country level. Am. J. Trop. Med. Hyg. 77 (suppl. 6), 133-135 (2007).

14. Gujjar, R. et al. Identification of a metabolically stable triazolopyrimidine-based dihydroorotate dehydrogenase inhibitor with antimalarial activity in mice. J. Med. Chem. 52, 1864-1872 (2009).

15. Patel, V. et al. Identification and characterization of small molecule inhibitors of Plasmodium falciparum dihydroorotate dehydrogenase. J. Biol. Chem. 283, 35078-35085 (2008).

16. Weissbuch, I. \& Leiserowitz, L. Interplay between malaria, crystalline hemozoin formation, and antimalarial drug action and design. Chem. Rev. 108, 4899-4914 (2008).

17. Pisciotta, J. M. et al. The role of neutral lipid nanospheres in Plasmodium falciparum haem crystallization. Biochem. J. 402, 197-204 (2007).

18. Sijwali, P. S. \& Rosenthal, P. J. Gene disruption confirms a critical role for the cysteine protease falcipain-2 in hemoglobin hydrolysis by Plasmodium falciparum. Proc. Natl Acad. Sci. USA 101, 4384-4389 (2004).

19. Sijwali, P. S., Koo, J., Singh, N. \& Rosenthal, P. J. Gene disruptions demonstrate independent roles for the four falcipain cysteine proteases of Plasmodium falciparum. Mol. Biochem. Parasitol. 150, 96-106 (2006).

20. Crowther, G. J. et al. Buffer optimization of thermal melt assays of Plasmodium proteins for detection of small-molecule ligands. J. Biomol. Screen. 14, 700-707 (2009).

21. Witola, W. H. et al. Disruption of the Plasmodium falciparum PfPMT gene results in a complete loss of phosphatidylcholine biosynthesis via the serinedecarboxylase-phosphoethanolamine-methyltransferase pathway and severe growth and survival defects. J. Biol. Chem. 283, 27636-27643 (2008).

22. Krnajski, Z. et al. Thioredoxin reductase is essential for the survival of Plasmodium falciparum erythrocytic stages. J. Biol. Chem. 277, 25970-25975 (2002).

23. McFadden, G. I. \& Roos, D. S. Apicomplexan plastids as drug targets. Trends Microbiol. 7, 328-333 (1999).

24. Reynolds, M. G. \& Roos, D. S. A biochemical and genetic model for parasite resistance to antifolates. Toxoplasma gondii provides insights into pyrimethamine and cycloguanil resistance in Plasmodium falciparum. J. Biol. Chem. 273, 3461-3469 (1998)

25. Lipinski, C. A., Lombardo, F., Dominy, B. W. \& Feeney, P. J. Experimental and computational approaches to estimate solubility and permeability in drug discovery and development settings. Adv. Drug Deliv. Rev. 46, 3-26 (2001)

26. Shenai, B. R. et al. Structure-activity relationships for inhibition of cysteine protease activity and development of Plasmodium falciparum by peptidyl vinyl sulfones. Antimicrob. Agents Chemother. 47, 154-160 (2003).

27. Ritz, C. \& Streibig, J. C. Bioassay analysis using R. J. Stat. Softw. 12, 22 (2005).

Supplementary Information is linked to the online version of the paper at www.nature.com/nature.

Acknowledgements This work was supported by the American Lebanese Syrian Associated Charities (ALSAC) and St Jude Children's Research Hospital (SJCRH, R.K.G.), the Medicines for Malaria Venture (W.C.V.V. and V.M.A.), National Institute of Allergy and Infectious Diseases (AI772682 (P.H.D.), AI075517 (R.K.G.), Al067921 (W.C.V.V.) and Al080625 (W.C.V.V.), AI28724 (D.S.R.), Al53862 (J.L.D.), Al35707 (P.J.R.), Al053680 (M.A.P. and P.K.R.), AI075594 (M.A.P., P.K.R. and I.B.), AI082617 (P.K.R.) and AI045774 (D.J.S.)), the National Cancer Institute (CA78039 (J.S.L.)), the Welch Foundation (I-1257 (M.A.P.)), the Doris Duke Charitable Foundation (P.J.R.), and the Ellison Medical Foundation (D.S.R.). We acknowledge A. B. Vaidya for providing the parasite strain D10_yDHOD. We acknowledge M. Sigal for assistance in the early leads project coordination, the SJCRH High Throughput Screening Center, particularly J. Cui; the SJCRH Lead Discovery Informatics Center, and the SJCRH High Throughput Analytical Chemistry Center, particularly C. Nelson and A. Lemoff; at UW, F. Buckner, W. Hol and A. Napuli (AI067921, W. Hol); S. Wei and W. Hao in the UT Southwestern HTS Center; and the Australian Red Cross Blood Service for the provision of $\mathrm{O}+$ erythrocytes to Griffith University.

Author Contributions W.A.G. and R.K.G. designed and coordinated the project. A.A.S. wrote the algorithms for the data analysis and generated the figures. Assays were conceived, performed and analysed by W.A.G. and D.B. (P. falciparum phenotypic screen), M.C. (human cell lines), D.C.S. (T. brucei), P.H.D. and D.S.R. (T. gondii), J.S.L. and E.R.S. (L. major), A.K.T. and D.J.S. (haemozoin inhibition), G.J.C. and W.C.V.V. (thermal melt experiments), M.A.P., P.K.R., F.E.M. and I.B. (PfDHOD), J.W.F. and P.K.R. (P. falciparum dihydrofolate reductase), J.G. and P.J.R. (PfFP-2), I.F. and M.K.R. (cytochrome $b c_{1}$ ), J.C. (P. falciparum mutant drug sensitivity). E.B.W., S.D., J.L.D. and V.M.A. (independent antimalarial in vitro experiments), F.Z. (in vitro pharmacokinetics), M.B.J.D., M.S.M., I.A.-B. and S.F. (in vivo pharmacokinetics and efficacy), I.B. (coordination of technology development and network development), S.C. and P.L.M. (re-synthesis). W.A.G., A.A.S. and R.K.G. wrote the manuscript. All authors contributed to the design of the experiments and the preparation of the manuscript.

Author Information Reprints and permissions information is available at www.nature.com/reprints. The authors declare no competing financial interests. Readers are welcome to comment on the online version of this article at www.nature.com/nature. Correspondence and requests for materials should be addressed to R.K.G. (kip.guy@stjude.org). 